\title{
FINITE $p$-SOLUBLE GROUPS WITH IRREDUCIBLE MODULAR REPRESENTATIONS OF GIVEN DEGREES
}

\author{
by J. F. HUMPHREYS \\ (Received 30th April 1975)
}

Let $G$ be a finite group, $p$ be a prime and $K$ be a field of characteristic p. Let

$$
K(G)=B_{1} \oplus \cdots \oplus B_{t}
$$

be a decomposition of the group ring of $G$ over $K$ as a sum of indecomposable two-sided ideals. An irreducible $K(G)$-module is said to be in the block $B_{i}$ if it occurs as a composition factor of $B_{i}$. The block containing the trivial $K(G)$-module is called the principal block of $G$.

Let $I$ be a subset of the positive integers with $1 \in I$. We denote by $\mathfrak{X}(I)$ (or by $\mathfrak{X}$ when $I$ is fixed) the class of finite $p$-soluble groups $G$ such that the dimension of every irreducible $K(G)$-module is in $I$, and by $\mathfrak{X}_{1}(I)$ (or by $X_{1}$ ) the class of finite $p$-soluble groups $G$ such that the dimension of each irreducible $K(G)$-module in the principal block of $G$ is in $I$. The object of this note is to investigate the relationship between $\mathfrak{X}(I)$ and $\mathfrak{X}_{1}(I)$, and to calculate these classes in some simple cases. Denoting by $O_{P}(G)$ the largest normal $p$-subgroup of a group $G$, and by $F_{p}(G)$ the largest normal subgroup of $G$ which has a normal $p$-complement, we can now state our main result.

Theorem 1. Let I be a subset of the positive integers with $1 \in I$. Let $\mathfrak{X}(I)$ and $\mathfrak{X}_{1}(I)$ be as defined above. Then

(i) $\mathfrak{X}(I)=\left\{G \mid G / O_{p}(G) \cong H / F_{p}(H)\right.$ for some $\left.H \in \mathfrak{X}_{1}(I)\right\}$ and

(ii) $\mathfrak{X}_{1}(I)=\left\{G \mid G / F_{p}(G) \in \mathfrak{X}(I)\right\}$.

Proof. (i) Let $T$ be an irreducible $K(G)$-module and suppose $G / O_{p}(G) \cong H / F_{p}(H)$ with $H \in \mathfrak{X}_{1}$. By Lemma 1.2 of (2), the kernel of $T$ contains $O_{p}(G)$ and so we may regard $T$ as a $K(H)$-module whose kernel contains $F_{p}(H)$. Lemma 2.3 of (2) now gives that $T$ is in the principal block of $K(H)$. (In fact Lemma 2.3 of (2) is proved under the assumption that $K$ is algebraically closed, but the techniques of (2) yield the general result quite easily). Thus $\operatorname{dim} T \in I$ and so $G \in \mathfrak{X}$.

Conversely, let $G \in \mathfrak{X}$ and define $M=G / O_{p}(G)$. Since every irreducible $K(M)$-module may be regarded as an irreducible $K(G)$-module, $M \in \mathfrak{X}$. By construction $O_{p}(M)=1$, so by Satz VI.7.20 of (4) there is a faithful, completely reducible $K(M)$-module $B$, say, over the field with $p$ 
elements. Let $H$ be the semi-direct product $M B$. A standard argument as used in the proof of (4: VI.7.24), for example, shows that $F_{p}(H)=B$.

We have thus constructed a group $H$ with

$$
H / F_{p}(H) \cong M B / B \cong M=G / O_{p}(G) .
$$

By Lemma 2.3 of (2), each irreducible $K(H)$-module in the principal blocks of $H$ has $F_{p}(H)$ in its kernel, and so may be regarded as an irreducible $K(G)$-module. Since $G \in \mathfrak{X}$, we have that $H \in \mathfrak{X}_{1}$.

(ii) By definition, a $p$-soluble group $G$ is in $\mathfrak{X}_{1}$ if and only if the dimension of each irreducible $K(G)$-module in the principal block of $G$ is in $I$. In view of Lemma 2.3 of (2), this is equivalent to saying that the dimension of each irreducible $K(G)$-module whose kernel contains $F_{p}(G)$ is in $I$. Thus $G \in \mathfrak{X}_{1}$ if and only if the dimension of each irreducible $K\left(G / F_{p}(G)\right)$-module is in $I$, that is if and only if $G / F_{p}(G)$ is in $\mathfrak{X}$.

Corollary 1. With the notation of the theorem, $\mathfrak{X}(I)$ is a formation if and only if $\mathfrak{X}_{1}(I)$ is a saturated formation.

Proof. If $\mathfrak{X}_{1}(I)$ is a saturated formation, Theorem $1(\mathrm{i})$ together with Hilfssatz VI.7.24 of (4) give that $\mathfrak{X}(I)$ is a formation.

Conversely, suppose that $\mathfrak{X}(I)$ is a formation. Theorem 1 (ii) implies that a group $G$ is in $\mathfrak{X}_{1}(I)$ if and only if $G / F_{p}(G) \in \mathfrak{X}(I)$ and $G / F_{q}(G)$ is a $p$-soluble group for $q \neq p$. Thus $\mathfrak{X}(I)$ is a locally defined formation and so is saturated by the main theorem of Gaschütz (see (4; VI.7.5)).

Corollary 2. With the notation of the theorem,

(i) for $G \in \mathfrak{X}_{1}(I)$ and $N$ any normal subgroup of $G, G / N \in \mathfrak{X}_{1}(I)$,

(ii) $G \in \mathfrak{X}_{1}(I)$ if and only if $G / \phi(G) \in \mathfrak{X}_{1}(I)$.

Proof. Let $H \in \mathfrak{X}(I)$ and $L$ be a normal subgroup of $H$. Since each irreducible $K(H / L)$-module is an irreducible $K(H)$-module, $H / L \in \mathfrak{X}(I)$. Thus $\mathfrak{X}(I)$ is closed under epimorphic images. The corollary can now be deduced from a proof of the theorem of Gaschütz referred to above.

In view of Corollaries 1 and 2 , it is natural to ask if, given $I$, every finite p-soluble group has $\mathfrak{X}_{1}(I)$-projectors. This is not the case, as is shown by the following.

Example 1. Let $N_{1}$ be elementary of order 9 , and $Q_{1}$ be a subgroup of $G L(2,3)$ isomorphic to the quaternion group of order 8 . Let $G_{1}$ be the semi-direct product of $N_{1}$ by $Q_{1}$ with the natural action of $Q_{1}$ on $N_{1}$. Let $G_{2} \cong G_{1}$ be the semi-direct product of $N_{2}\left(\cong N_{1}\right)$ by $Q_{2}\left(\cong Q_{1}\right)$ and let $G=G_{1} \times G_{2}$.

Let $K$ be an algebraically closed field of characteristic 3 and $I=\{1,2\}$. Then $G_{i} \in \mathfrak{X}(I)$ for $i=1,2$ but $G \notin \mathfrak{X}(I)$ since there is a 4 dimensional irreducible $K(G)$-module. Also $G$ has just one block (since $O_{3^{\prime}}(G)=1$ ), so 
$G \notin \mathfrak{X}_{1}(I)$. However $G / N_{i} \in \mathfrak{X}_{1}(I)(i=1,2)$ since

$$
G / N_{1} \cong G_{2} \times Q_{1} \cong G / N_{2} \text {. }
$$

Suppose that $G$ has an $\mathfrak{X}_{1}(I)$-projector $F$. Then $G=F N_{1}$ and so $F \cap N_{1}$ is a normal subgroup of $G$, giving that $F \cap N_{1}=1$ by the minimality of $N_{1}$. Hence a Sylow 3-subgroup $N$ of $F$ has order 9 and $N$ is normalized by $F$ and by $N_{1}$ since $N_{1} \times N_{2}$, the unique Sylow 3-subgroup of $G$, is abelian. Hence $N$ is a normal subgroup of $G$. Since the non-identity elements of $N_{i}$ are permuted transitively by the elements of $Q_{i}(i=1,2)$, it follows easily that $N_{1}$ and $N_{2}$ are the only two minimal normal subgroups of $G$. Thus $N=N_{2}$ and $F N_{2}=F \neq G$. This shows that $G$ has no $\mathfrak{X}_{1}(I)$-projectors.

As our first application of Theorem 1 , we consider the situation where $k$ is the field with $p$ elements, $n$ is a positive integer and $I_{n}$ is the set of positive integers which divide $n$.

Theorem 2. Let $n$ be a positive integer and $G$ be a group of order coprime to $n$. Then $G \in \mathfrak{X}\left(I_{n}\right)$ if and only if $G / O_{p}(G)$ is abelian of exponent dividing $p^{n}-1$.

Proof. Suppose $G \in \mathfrak{X}\left(I_{n}\right)$ and $V$ is an irreducible $k(G)$-module with $N=\operatorname{ker} V$. By (4: VI.8.1), $G / N$ is cyclic of order dividing $p^{n}-1$. Since the intersection of the kernels of the irreducible $k(G)$-modules is $O_{p}(G)$, we have that $G / O_{p}(G)$ is abelian of exponent dividing $p^{n}-1$.

Conversely, suppose that $G / O_{p}(G)$ is abelian of exponent dividing $p^{n}-1$ and that $V$ is an irreducible $k(G)$-module. It follows by (4:: II.3.10) that the dimension of $V$ divides $n$.

Recall that for any $p$-soluble group $G$, the arithmetic $p$-rank of $G$ is defined to be the lowest common multiple of the $k$-dimensions of the $p$-chief factors of $G$.

Corollary 3. Let $n$ be a positive integer and $G$ be a group of order cop rime to $n$. Then $G$ has arithmetic $p$-rank dividing $n$ if and only if $G \in \mathfrak{X}_{1}\left(I_{n}\right)$.

Proof. By Theorem 1, Theorem 2 and (4: VI.8.3).

For our second application, denote by $\bar{Q}$ the algebraic closure of the field $\boldsymbol{Q}$ of rational numbers. Choose a fixed extension $\nu_{p}$ of the $p$-adic exponential valuation of $\boldsymbol{Q}$ to $\overline{\boldsymbol{Q}}$. Let $R$ denote the local ring of $\nu_{p}$ in $\overline{\boldsymbol{Q}}, \boldsymbol{P}$ denote the corresponding prime ideal, and $K=R / P$. Let $I_{p^{\prime}}$ be the set of positive integers coprime to $p$. We then have

Lemma 2. Each factor group and each normal subgroup of a group in $\mathfrak{X}\left(I_{p^{\prime}}\right)$ is also in $\mathfrak{X}\left(I_{p^{\prime}}\right)$.

Proof. Let $G \in \mathfrak{X}\left(I_{p^{\prime}}\right)$ and suppose $N$ is a normal subgroup of $G$. By Mackey's subgroup theorem (4: V.16.9), an irreducible $K(N)$-module $T$ occurs as a component of $\left(T^{G}\right)_{N}$. However each composition factor of $T^{G}$ 
has dimension coprime to $p$ and so by Clifford's theorem (4: V.17.3), the irreducible components of $\left(T^{G}\right)_{N}$ have dimension coprime to $p$.

The result for factor groups is trivial.

Theorem 3. A p-soluble group $G$ is in $\mathfrak{X}\left(I_{p^{\prime}}\right)$ if and only if $G$ has a normal Sylow p-subgroup.

Proof. Suppose $G$ has a normal Sylow $p$-subgroup $N$. Then each irreducible $K(G)$-module may be regarded as an irreducible $K(G / N)$ module and so has dimension coprime to $p$.

We prove the converse implication by induction on $|G|$. Let $N$ be a maximal normal subgroup of $G$, so that $M \in \mathfrak{X}$ by Lemma 2 . By induction, $M$ has a normal Sylow $p$-subgroup $N$. If $p$ is coprime to $|G: M|, N$ is a normal Sylow $p$-subgroup of $G$. Thus by the maximality of $N$, we may suppose that $|G: M|=p$. If $N \neq 1$, induction applied to $G / N$ gives the result, so we may suppose that $|M|$ is coprime to $p$.

Since $G$ has a normal $p$-complement and abelian Sylow $p$-subgroups, we can apply a result of Richen (5), to deduce that the restriction of the character of every irreducible $\bar{Q}(G)$-module to the $p$-regular conjugacy classes of $G$ is a Brauer irreducible character of $G$. Thus each irreducible $\bar{Q}(G)$-module has dimension coprime to $p$ and so, by a result of Fong (1: 3D), $G$ has a normal Sylow $p$-subgroup as required.

Corollary 4. A p-soluble $G$ is in $\mathfrak{X}_{1}\left(I_{p^{\prime}}\right)$ if and only if $G$ has $p$-length 1.

Proof. By Theorem 1 and Theorem 3.

Remark. Theorem 3 is a modular analogue of Lemma $3 D$ of (1) and Corollary 4 is an analogue of Theorem $3 \mathrm{E}$ of (1).

As a final application, we consider the case where $I$ is a finite set.

Theorem 4. There exists an integer valued function $f(p, m, n)$ so that for any finite p-soluble group $G$ whose Sylow p-subgroups have order at most $p^{m}$ with the property that each irreducible $K(G)$-module in the principal block of $G$ has dimension at most $n$, there exists a normal subgroup $N$ of $G$ of index at most $f(p, m, n)$ such that $N^{\prime}$ has a normal p-complement.

Proof. By Theorem 1 and the theorem of (3).

Example 2. Let $G=S_{5}$, the symmetric group on 5 symbols, so that $F_{3}(G)=1$. Let $K$ be algebraically closed of characteristic 3 and $I$ be the set of integers coprime to 3 . Since $G$ has two irreducible $K(G)$-modules in its principal block, of degrees 1 and $4, G \in \mathfrak{X}_{1}$. However $G \notin \mathfrak{X}$ since there is an irreducible $K(G)$-module of dimension 6. This example shows that neither (i) nor (ii) of Theorem 1 holds for arbitrary finite groups. 


\section{REFERENCES}

(1) P. FONG, On the characters of p-soluble groups, Trans. Amer. Math. Soc. 98 (1961), 263-284.

(2) P. FONG and W. GASCHÜTZ, A note on modular representations of solvable groups, J. Reine Angew. Math. 208 (1961), 73-78.

(3) J. F. HUMPhREYS, Groups with modular irreducible representations of bounded degree, J. London Math. Soc. 5 (1972), 233-234.

(4) B. HUPPERT, Endliche Gruppen I. (Springer-Verlag, Berlin, 1967).

(5) F. Richen, Decomposition numbers of p-solvable groups, Proc. Amer. Math. Soc. 25 (1970), 100-104.

THE UNIVERSITY

LIVERPOOL 